\title{
Immunohistochemical detection of human herpes virus-8 latent nuclear antigen-1 is useful in the diagnosis of Kaposi sarcoma
}

\author{
Rajiv M Patel, John R Goldblum and Eric D Hsi \\ Division of Pathology and Laboratory Medicine, The Cleveland Clinic Foundation, Cleveland, OH, USA
}

\begin{abstract}
Kaposi sarcoma is a low-grade vascular neoplasm that has been shown by molecular analysis to uniformly express the latent nuclear antigen-1 of human herpes virus 8. Differentiating Kaposi sarcoma from other benign or malignant vascular tumors, as well as other nonvascular spindle cell soft-tissue neoplasms, can be challenging. Thus, detection of human herpes virus 8 in fixed tissues would be diagnostically useful. Recently, a monoclonal antibody to human herpes virus 8 latent nuclear antigen-1 has become commercially available for immunohistochemical analysis. We sought to study the sensitivity and specificity of this antibody in the detection of human herpes virus 8 latent nuclear antigen-1 in Kaposi sarcoma. Fixed, paraffin-embedded tissue sections from 21 cases of Kaposi sarcoma, nine cases of spindle cell hemangioma, five cases of cutaneous angiosarcoma, five cases of dermatofibrosarcoma protuberans, one case of vascular transformation of a lymph node, four cases of pilar leiomyoma, four cases of stasis dermatitis, four cases of pyogenic granuloma, and three cases of spindled melanoma were examined immunohistochemically using the rat monoclonal antibody to human herpes virus 8 latent nuclear antigen-1, open reading frame-73 (Advanced Biotechnologies Inc.). Tissue sections were stained with automated immunostainers (Ventana) using heat-induced epitope retrieval and a standard DAB detection kit (Ventana) modified to detect rat Ab. Strong, diffuse, nuclear staining in $>10 \%$ of tumor cells was considered a positive result. In all, 21/21 cases of Kaposi sarcoma showed strong, diffuse, nuclear staining for human herpes virus 8 latent nuclear antigen-1 $(100 \%)$, whereas all cases of spindle cell hemangioma, cutaneous angiosarcoma, dermatofibrosarcoma protuberans, vascular transformation of lymph node, pilar leiomyoma, stasis dermatitis, pyogenic granuloma, and spindled melanoma were negative for this antigen. The monoclonal antibody to human herpes virus 8 latent nuclear antigen-1, open reading frame-73, is a highly sensitive and specific marker of human herpes virus 8 infection in paraffin-embedded tissue sections of Kaposi sarcoma. As such, it is an extremely useful tool for differentiating between Kaposi sarcoma and other vascular and nonvascular spindle cell lesions, which do not express human herpes virus 8 latent nuclear antigen-1.

Modern Pathology (2004) 17, 456-460, advance online publication, 27 February 2004; doi:10.1038/modpathol.3800061
\end{abstract}

Keywords: HHV-8, immunohistochemistry; Kaposi sarcoma; differential diagnosis

Kaposi sarcoma is a low-grade vascular neoplasm first described by Kaposi ${ }^{1}$ in 1872. Four clinical forms have been described: classic Kaposi sarcoma is seen in males of Mediterranean or Eastern European origin. African (endemic) Kaposi sarcoma occurs in younger adults and children in Central Africa. Epidemic (HIV-associated) Kaposi sarcoma is found in immunosuppressed patients with human immunodeficiency virus infection. Kaposi sarcoma associated with immunosuppressive therapy is

Correspondence: ED Hsi, MD, Department of Clinical Pathology, L-11, Cleveland Clinic Foundation, 9500 Euclid Avenue, Cleveland, OH 44195, USA.

E-mail: hsie@ccf.org

Received 19 February 2003; revised 9 October 2003; accepted 23 December 2003; published online 27 February 2004 found in patients treated for transplant rejection. In 1994, Chang et al discovered a herpes-like virus in the Kaposi sarcoma cells of a patient with AIDS. ${ }^{2}$ The virus is now most widely referred to as Kaposi's sarcoma-associated herpes virus (KSHV) or human herpes virus 8 (HHV-8). ${ }^{3}$ It has subsequently been shown to be present in essentially all cases of Kaposi sarcoma ${ }^{4}$ as well as other conditions including primary effusion lymphoma, ${ }^{5-7}$ some cases of multicentric Castleman's disease, ${ }^{7-10}$ reactive angioendotheliomatosis, ${ }^{11}$ and in the recently described plasmablastic lymphoproliferative disorders arising in the setting of multicentric Castleman's disease. ${ }^{12}$

Distinguishing Kaposi sarcoma from other benign or malignant vascular tumors, as well as other nonvascular spindle cell soft-tissue neoplasms, can 
be challenging. The differential diagnosis may include cutaneous angiosarcoma, spindle cell hemangioma, dermatofibrosarcoma protuberans, vascular transformation of lymph nodes, pilar leiomyoma, stasis dermatitis, pyogenic granuloma, and spindled melanoma among others. Thus, immunohistochemical detection of HHV-8 in fixed tissues would be diagnostically useful, enabling one to differentiate Kaposi sarcoma from these entities. Recently, a monoclonal antibody to HHV-8 latent nuclear antigen-1 (LNA-1), open reading frame-73 (ORF-73), has become commercially available, which is suitable for immunohistochemistry in fixed tissues.

HHV-8 LNA-1 is a protein encoded for by ORF-73 of the virus' genome. The protein is expressed predominantly during viral latency and appears to play a role in viral integration into the host genome. ${ }^{13,14}$ It also interferes in apoptosis via interactions with p53. ${ }^{15}$ Antibodies to LNA-1 have been used in formalin-fixed, paraffin-embedded tissues previously. ${ }^{9,11,12,16,17}$ At the time of this writing, we know of no study evaluating the utility of HHV-8 LNA-1, ORF-73 antibodies in distinguishing Kaposi sarcoma from other histologically similar vascular and nonvascular spindle cell neoplasms. For this reason, we sought to study the sensitivity and specificity of this antibody in the detection of HHV-8 LNA-1 in Kaposi sarcoma.

\section{Materials and methods}

In total, 21 cases of Kaposi sarcoma (Table 1), nine cases of spindle cell hemangioma, five cases of cutaneous angiosarcoma from four patients, five

Table 1 Characteristics of Kaposi sarcoma lesions

\begin{tabular}{rllll}
\hline Case number & Site & Stage & Age & Sex \\
\hline 1 & & & & \\
2 & Skin, left arm & Patch/plaque & 77 & $\mathrm{M}$ \\
3 & Skin, thigh & Patch/plaque & 50 & $\mathrm{M}$ \\
4 & Skin, right foot & Patch/plaque & 46 & $\mathrm{M}$ \\
5 & Skin, chest & Patch/plaque & 51 & $\mathrm{M}$ \\
6 & Skin, back & Patch/plaque & 63 & $\mathrm{M}$ \\
7 & Skin, shoulder & Patch/plaque & 44 & $\mathrm{M}$ \\
8 & Skin, shoulder & Patch/plaque & 47 & $\mathrm{M}$ \\
9 & Skin, flank & Patch/plaque & 59 & $\mathrm{M}$ \\
10 & Skin, arm & Patch/plaque & 36 & $\mathrm{M}$ \\
11 & Skin, foot & Patch/plaque & 39 & $\mathrm{M}$ \\
12 & Skin, forehead & Nodular & 46 & $\mathrm{M}$ \\
13 & Skin, ear & Nodular & 54 & $\mathrm{M}$ \\
14 & Skin, foot & Nodular & 87 & $\mathrm{M}$ \\
15 & Lymph node & NA & 42 & $\mathrm{M}$ \\
16 & Trachea & NA & 38 & $\mathrm{M}$ \\
17 & Lung & NA & 39 & $\mathrm{M}$ \\
18 & Stomach & NA & 41 & $\mathrm{M}$ \\
19 & Stomach & NA & 59 & $\mathrm{M}$ \\
20 & Duodenum & NA & 43 & $\mathrm{M}$ \\
21 & Rectum & NA & 46 & $\mathrm{M}$ \\
& Soft tissues, leg & NA & 83 & $\mathrm{M}$ \\
\hline
\end{tabular}

NA: not applicable, M: Male. cases of dermatofibrosarcoma protuberans, one case of vascular transformation of a lymph node, four cases of pilar leiomyoma, four cases of stasis dermatitis, four cases of pyogenic granuloma, and three cases of spindled melanoma were studied. The H\&E sections of each case were examined and the diagnoses confirmed by a soft-tissue pathologist (JRG). Fixed, paraffin-embedded tissue sections were then examined immunohistochemically using the rat monoclonal antibody to HHV-8 LNA-1, ORF73 (Advanced Biotechnologies Inc.). Tissue sections were stained with an automated immunostainer (Ventana ES) using heat-induced epitope retrieval and standard DAB detection kit (Ventana) modified to detect rat $\mathrm{Ab}$ (three drops rabbit normal serum concentrate, one drop biotinylated rabbit, anti-rat secondary antibody (Vector Laboratories, Burlingame, CA, USA) for every $10 \mathrm{ml}$ of biotinylated immunoglobulin from the standard DAB detection kit). Primary antibody dilution was 1:1000 with an incubation time of $32 \mathrm{~min}$. Antigen retrieval was achieved with a 15-min treatment in a microwave pressure cooker with citrate buffer, followed by a 15min cool down. Strong, diffuse, nuclear staining in $>10 \%$ of the tumor cells was considered a positive result. A cell block of the primary effusion lymphoma cell line BC-3 and a case of Kaposi sarcoma were used as positive controls.

\section{Results}

Characteristic histologic features of Kaposi sarcoma included spindle-shaped tumor cells surrounding hyperemic vascular slits, often accompanied by extravasated erythrocytes, hemosiderin, and fibrosis. The sites of the 21 Kaposi sarcoma specimens are summarized in Table 1. Similar to Kaposi sarcoma, cases of spindle cell hemangioma were composed of bland spindle cell proliferations between vascular lumens with extravasated erythrocytes. However, unlike Kaposi sarcoma, vacuolated cells were sometimes noted lining lumens, as well as epithelioid endothelial cells. Five cutaneous angiosarcomas, two spindled, one epithelioid, and two mixed, were studied from four patients. Infiltrating, anastomosing vascular channels lined by numerous plump spindled-to-epithelioid cells with large hyperchromatic nuclei characterized these cases. Infiltrative lesions with bland spindle cells in a tight, storiform pattern, were the rule for cases of dermatofibrosarcoma protuberans. The lymph node with vascular transformation demonstrated conversion of nodal sinuses into numerous capillary-like spaces containing some erythrocytes. Pilar leiomyomas were composed of nodular, dermal aggregates of poorly circumscribed, intersecting fascicles of eosinophilic spindle cells with plump, cigar-shaped nuclei. The cases of stasis dermatitis all had a superficial dermal vascular proliferation within a background of dermal fibrosis, perivascular 
lymphohistiocytic infiltrates, extravasated erythrocytes, and hemosiderophages. There was variable acanthosis and hyperkeratosis. All pyogenic granulomas were nodular proliferations of small capillaries with epidermal ulceration, resembling granulation tissue. Finally, the spindled melanomas had spindled cells with highly atypical nuclei and scant cytoplasm embedded in a fibrotic dermal stroma; cellularity varied.

The results of the immunohistochemical analysis are summarized in Table 2. All 21 Kaposi cases showed strong, nuclear staining for HHV-8 (100\%). Cutaneous Kaposi sarcoma cases included 10 patch/ plaque and three nodular lesions. Nodular lesions generally had more positive spindle cells compared to patch/plaque stage lesions. Patch/plaque stage lesions demonstrated staining in $10 \%$ of the nuclei of spindled/fusiform cells in one case, $20 \%$ in four cases, $40 \%$ in two cases, $50 \%$ in two cases, and $75 \%$ in one case (Figure 1a). Nodular stage lesions had $40 \%$ positive nuclei in one case, and $50 \%$ positive nuclei in two cases (Figure 1b). Non-cutaneous Kaposi sarcoma cases included one mucosal biopsy specimen each from the trachea, duodenum, and rectum, two biopsy specimens from the stomach, an endobronchial biopsy of the lung, and a resection specimen from the soft tissues of the leg. Staining was seen in 20 and $40 \%$ of nuclei in the two cases of gastric Kaposi sarcoma, $50 \%$ of nuclei in the tracheal, lung, and rectal cases, $75 \%$ of nuclei in the duodenal case, and $90 \%$ of nuclei in the softtissue Kaposi sarcoma (Figure 1c). Importantly, normal vascular endothelial cells were negative in all cases of Kaposi sarcoma. The spindle cell hemangiomas (9), cutaneous angiosarcomas (5), dermatofibrosarcoma protuberans (5), the vascular transformation of a lymph node case (1), pilar leiomyomas (4), cases of stasis dermatitis (4), pyogenic granulomas (4), and spindled melanomas (3) were all negative for this antigen (Figure 1d, Table 2). Of note was the occasional light background staining of eccrine structures and keratinocytes in the skin. (After performance of this study, our clinical laboratory switched from the Ventana ES to the Ventana Benchmark automated immuno-

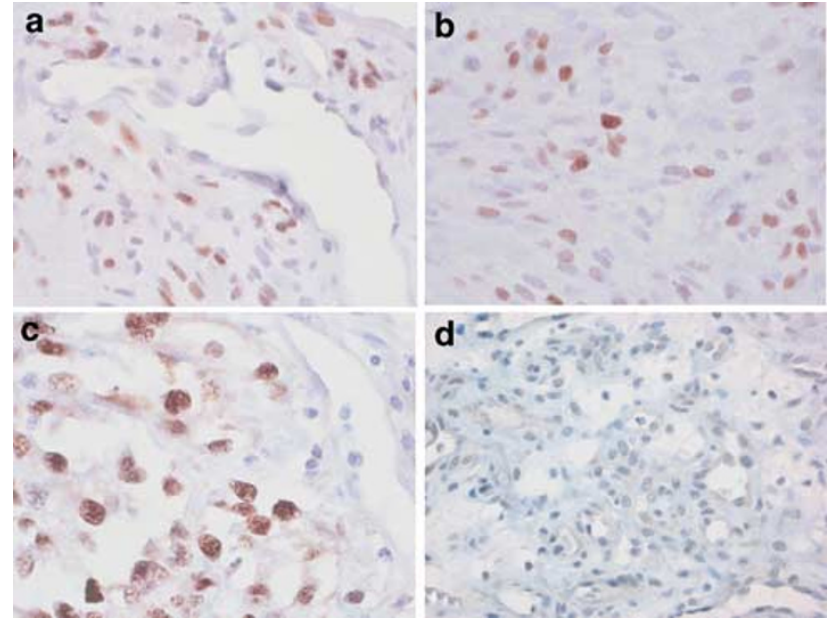

Figure 1 Representative high-power views of immunohistochemical staining for HHV-8 LNA-1 in (a), cutaneous patch/plaque Kaposi sarcoma, (b) cutaneous nodular Kaposi sarcoma; and (c), soft-tissue Kaposi sarcoma. Note that all normal vascular endothelial cells are negative for HHV-8 LNA-1. (d) Representative case demonstrating the absence of reactivity for HHV-8 LNA-1 in a case of pyogenic granuloma.

stainer. The new protocol has eliminated background staining.)

\section{Discussion}

In their original paper, Chang et $a l^{2}$ used representational difference analysis (RDA) to analyze 27 cases of AIDS-associated Kaposi sarcoma and 142 nonKaposi sarcoma cases. They discovered unique DNA sequences in $90 \%$ of AIDS-associated Kaposi sarcoma. ${ }^{2}$ Using polymerase chain reaction (PCR), Moore et $a l^{4}$ studied 11 cases of AIDS-related, six cases of classic, and four cases of non-HIV-associated Kaposi sarcoma, along with 21 negative controls for the presence of these sequences. PCR product was found in 20 of 21 (95\%) tissue samples from the patients with Kaposi sarcoma and only one of 21 control samples (5\%). Subsequently, these sequences were found by Moore et $a l^{18}$ to belong to a new human herpes virus, now designated HHV-8.

Table 2 Immunostaining results summary

\begin{tabular}{|c|c|c|c|c|}
\hline Tumor type & $H H V-8$ positive & HHV-8 negative & Total & Percent \\
\hline Kaposi’s sarcoma & 21 & 0 & 21 & 100 \\
\hline Spindle cell hemangioma & 0 & 9 & 9 & 0 \\
\hline Angiosarcoma & 0 & 5 & 5 & 0 \\
\hline Dermatofibrosarcoma protuberans & 0 & 5 & 5 & 0 \\
\hline Vascular transformation of lymph node & 0 & 1 & 1 & 0 \\
\hline Pilar leiomyoma & 0 & 4 & 4 & 0 \\
\hline Stasis dermatitis & 0 & 4 & 4 & 0 \\
\hline Pyogenic granuloma & 0 & 4 & 4 & 0 \\
\hline \multirow[t]{2}{*}{ Spindled melanoma } & 0 & 3 & 3 & 0 \\
\hline & & & 56 & \\
\hline
\end{tabular}


Distinguishing Kaposi sarcoma from other benign or malignant vascular tumors as well as other nonvascular spindle cell soft-tissue neoplasms may, on occasion, be difficult. The histologic features of these lesions overlap, leading to diagnostic dilemmas. With the discovery of HHV-8 in all forms of Kaposi sarcoma, ${ }^{4}$ it became possible to consider virus detection as a potential diagnostic test. Molecular methods have traditionally been used to identify HHV-8 in human tissues. These include PCR amplification, 2,16,19,20 direct in situ hybridization, ${ }^{6,21}$ in situ $\mathrm{PCR},{ }^{22}$ and reverse transcriptase (RT) in situ PCR. ${ }^{23}$ These methods are labor intensive, time consuming, and require highly skilled laboratory personnel. In addition, highly sensitive PCR methodologies have led to a recent controversy. Initial studies using these methods suggested a biological association between HHV-8 and multiple myeloma. However, most experts now believe these to be 'false' positives attributed to overamplification of 'bystander' HHV-8 sequences commonly present in healthy individuals with a low rate of infection. ${ }^{24-26}$ Recent commercial availability of a monoclonal antibody to HHV-8 LNA-1 has made cost-effective, tissue-localized identification of HHV-8 in fixed human specimens possible. The reliable detection of HHV-8 in fixed tissues by immunohistochemistry could enable one to differentiate Kaposi sarcoma from other histologically similar entities. For this reason, we sought to study the sensitivity and specificity of this antibody in the detection of HHV-8 LNA-1 in Kaposi sarcoma.

All of our Kaposi sarcoma cases demonstrated strong, nuclear staining for HHV-8 (100\%), whereas all cases of spindle cell hemangioma (9), cutaneous angiosarcoma (5), dermatofibrosarcoma protuberans (5), vascular transformation in a lymph node (1), pilar leiomyoma (4), stasis dermatitis (4), pyogenic granuloma (4), and spindled melanoma (3) were negative for this antigen $(0 \%)$. The uniform expression of HHV-8 in all of our cases of Kaposi sarcoma $(100 \%$ sensitivity) confirms the observations of previous investigators using other methods.

Antibodies to HHV-8 LNA-1 have been used previously to identify HHV-8 by IHC. ${ }^{9,11,12,16,17}$ In a survey of HHV-8 + lesions, Dupin et $a l^{9}$ used an antibody to HHV-8 LNA-1 to study the distribution of cell types latently infected by HHV-8 in patch/ plaque and nodular Kaposi sarcoma, multicentric Castleman's disease, and primary effusion lymphoma. These authors studied 14 cases of Kaposi sarcoma and found nuclear staining in all stages of Kaposi sarcoma. They noted fewer positive cells in patch/plaque lesions compared to nodular lesions. No staining was found in normal endothelium. Our results are similar to Dupin et al, as we noted a similar pattern of staining with fewer immunoreactive spindle cells noted in patch/plaque lesions compared to nodular lesions. These authors also found LNA-1 expression in cells of other HHV-8 positive lesions such as multicentric Castleman's disease and primary effusion lymphoma, but no staining was found in samples of multiple myeloma, prostate cancer, or angiosarcoma. ${ }^{9}$ Unlike Dupin et al, we also studied examples of spindle cell lesions that might enter the differential diagnosis of Kaposi sarcoma (eg dermatofibrosarcoma protuberans), and found them to be uniformly negative for HHV-8 LNA-1. In a study of 13 cases of multicentric Castleman's disease using the same antibody, Du et $a l^{12}$ found that all the $13(100 \%)$ cases contained a majority of plasmablasts positive for HHV-8. Most recently, McMenamin et $a l^{11}$ reported that four of their cases of reactive angioendotheliomatosis stained positive for HHV-8 LNA in lesional cells. Interestingly, two of the four cases arose in immunosuppressed patients. Cool et $a l^{17}$ have also reported HHV-8 + cells both in and around plexiform lesions in a subset of cases of primary pulmonary hypertension. These results may expand the types of vascular lesions in which HHV-8 may be detected.

The high sensitivity and specificity of automated immunohistochemical detection for HHV-8 in Kaposi sarcoma with this antibody make it a reliable and cost-effective means of differentiating Kaposi sarcoma from other vascular and nonvascular spindle cell lesions. Furthermore, since it does not rely on amplification of nucleic acid and allows tissue localization, false-positive results or detection of 'bystander' virus may be less likely. We believe it to be an extremely useful tool to the surgical pathologist confronted with a lesion in which Kaposi sarcoma is a diagnostic consideration.

\section{Acknowledgements}

We thank Serhan Alkan, MD for providing the PEL, BC-3 cell block for a positive control.

\section{References}

1 Kaposi M. Idiopathic multiple pigmented sarcoma of the skin. CA Cancer J Clin 1982;32:340-347.

2 Chang Y, Cesarman E, Pessin M. Identification of herpesvirus-like DNA sequences in AIDS-associated Kaposi's sarcoma. Science 1994;266:1865-1869.

3 Antman K, Chang Y. Kaposi's sarcoma. N Engl J Med 2000;342:1027-1038.

4 Moore PS, Chang Y. Detection of herpes virus-like DNA sequences in Kaposi's sarcoma in patients with and those without HIV infection. $N$ Engl J Med 1995;332:1181-1185.

5 Uphoff CC, Carbone A, Gaidano G, et al. HHV-8 infection is specific for cell lines derived from primary effusion (body cavity-based) lymphomas. Leukemia 1998;12:1806-1809.

6 Said JW, Shintaku IP, Asou H, et al. Herpesvirus 8 inclusions in primary effusion lymphoma: report of a 
unique case with T-cell phenotype. Arch Pathol Lab Med 1999;123:257-260.

7 Said J. Kaposi's sarcoma-associated herpesvirus (KSHV): a new viral pathogen associated with Kaposi's sarcoma, primary effusion lymphoma, and multicentric Castleman's disease. West J Med 1997;167:37-38.

8 Teruya-Feldstein J, Zauber $\mathrm{P}$, Setsuda JE, et al. Expression of human herpesvirus-8 oncogene and cytokine homologues in an HIV-seronegative patient with multicentric Castleman's disease and primary effusion lymphoma. Lab Invest 1998;78:1637-1642.

9 Dupin N, Fisher C, Kellam P, et al. Distribution of human herpesvirus-8 latently infected cells in Kaposi's sarcoma, multicentric Castleman's disease, and primary effusion lymphoma. Proc Natl Acad Sci USA 1999;96:4546-4551.

10 Ascoli V, Signoretti S, Onetti-Muda A, et al. Primary effusion lymphoma in HIV-infected patients with multicentric Castleman's disease. J Pathol 2001;193: 200-209.

11 McMenamin ME, Fletcher CD. Reactive angioendotheliomatosis: a study of 15 cases demonstrating a wide clinicopathologic spectrum. Am J Surg Pathol 2002;26:685-697.

$12 \mathrm{Du}$ MQ, Liu H, Diss TC, et al. Kaposi sarcomaassociated herpesvirus infects monotypic (IgM lambda) but polyclonal naive B cells in Castleman disease and associated lymphoproliferative disorders. Blood 2001;97:2130-2136.

13 Cotter MA, Robertson ES. The latency-associated nuclear antigen tethers the Kaposi's sarcoma-associated herpesvirus genome to host chromosomes in body cavity-based lymphoma cells. Virology 1999;264:254-264.

14 Cotter MA, Robertson ES. Molecular biology of Kaposi's sarcoma-associated herpesvirus. Front Biosci 2002;7:d358-d375.

15 Friborg. Jr J, Kong W, Hottiger MO, et al. p53 inhibition by the LANA protein of KSHV protects against cell death. Nature 1999;402:889-894.

16 Huang Q, Chang KL, Gaal K, et al. Primary effusion lymphoma with subsequent development of a small bowel mass in an HIV-seropositive patient: a case report and literature review. Am J Surg Pathol 2002;26:1363-1367.

17 Cool CD, Rai PR, Yeager ME, et al. Expression of human herpesvirus 8 in primary pulmonary hypertension. N Engl J Med 2003;349:1113-1122.

18 Moore PS, Gao SJ, Dominguez G, et al. Primary characterization of a herpesvirus agent associated with Kaposi's sarcoma. J Virol 1996;70:549-558.

19 Cesarman E, Chang Y, Moore PS, et al. Kaposi's sarcoma-associated herpesvirus-like DNA sequences are present in AIDS-related body cavity based lymphomas. N Engl J Med 1995;332:1186-1191.

20 Nador RG, Cesarman E, Chadbum A. Primary effusion lymphoma: a distinct entity associated with the Kaposi-associated herpes virus. Blood 1996;88: 645-656.

21 Said JW, Rettig MR, Heppner K, et al. Localization of Kaposi's sarcoma-associated herpesvirus in bone marrow biopsy samples from patients with multiple myeloma. Blood 1997;90:4278-4282.

22 Hsi ED, Foreman KE, Duggan J, et al. Molecular and pathologic characterization of an AIDS-related body cavity-based lymphoma, including ultrastructural demonstration of human herpesvirus-8: a case report. Am J Surg Pathol 1998;22:493-499.

23 Wakely. Jr PE, Menezes G, Nuovo GJ. Primary effusion lymphoma: cytopathologic diagnosis using in situ molecular genetic analysis for human herpesvirus 8 . Mod Pathol 2002;15:944-950.

24 Azzi A, Fanci R, De Santis R, et al. Human herpesvirus 8 DNA sequences are present in bone marrow from HIV-negative patients with lymphoproliferative disorders and from healthy donors. $\mathrm{Br} \mathrm{J}$ Haematol 2001;113:188-190.

25 Zhu YX, Li ZH, Voralia M, et al. Antigenic open reading frames from HHV-8 are present in multiple myeloma patients and normal individuals at similar frequency. Leuk Lymphoma 2002;43:369-375.

26 Brander C, Raje N, O'Connor PG, et al. Absence of biologically important Kaposi sarcoma-associated herpesvirus gene products and virus-specific cellular immune responses in multiple myeloma. Blood 2002;100:698-700. 